\title{
Higgs Particle - End of Certain Story and What Next?
}

\author{
S. POKORSKI \\ Faculty of Physics, University of Warsaw, L. Pastera 5, 02-093 Warsaw, Poland \\ Doi: 10.12693/APhysPolA.139.222 \\ *e-mail: $\quad$ stefan.pokorski@fuw.edu.pl
}

\begin{abstract}
The significance of the discovery of the Higgs particle and open questions in particle physics are briefly reviewed.
\end{abstract}

topics: Higgs particle, symmetries

\section{Introduction}

In this paper, the physics of elementary interactions and its interface with cosmology is discussed. This was - not without reason - one of important topics of the jubilee congress of the Polish Physical Society (PPS) held on the occasion of its centenary. For more than seventy years now, Polish physicists, experimentalists and theorists have been significantly contributing, and with international recognition, to world research in this field of basic physics. This is confirmed by the fact that they maintain close scientific ties with the biggest international laboratories, such as CERN, DESY, FERMILAB, KAMIOKANDE, and many university groups all over the world.

\section{The story}

The story began in 1896 with the discovery of natural radioactivity by Henri Becquerel. It was an accidental discovery - made against the scientific atmosphere of these times which was reflected, for instance, in such remarks of great scholars of that period (quoted after Andrzej Kajetan Wróblewski, Historia fizyki, PWN) as:

- "Le monde est aujourd'hui sans mystère" (Marcellin Berthelot, Les Origines de l'Alchimie, 1885),

\section{or}

- "All fundamental laws of physics are already known" (Albert Michelson, a speech delivered at the University of Chicago during the opening of the Ryerson Laboratory, 1884).

The discovery of natural radioactivity has opened a new chapter in the physics of elementary interactions. With fundamental contributions from Maria Skłodowska-Curie, Pierre Curie and Ernest
Rutherford, it was discovered that the new phenomenon consists of two types of radiation, termed by Rutherford as $\alpha$ and $\beta$ radiation. Ultimately, they were understood as a result of two new elementary interactions, manifesting themselves only at extremely short length scales, that is at the quantum level. They are now known as strong and weak interactions. Together with the already well known earlier gravitational and electromagnetic ones, they complete the list of elementary forces known up to date.

As the front of research in the physics of elementary interactions moves on with time, it leaves behind more specialized branches of physics together with their strong impact on other sciences and applied research. The list of such branches is very long and includes, e.g., atomic physics, nuclear physics, condensed matter physics, interface with cosmology and astrophysics, as well as is linked to chemistry, biology, medicine, technical science, etc. The importance of basic research and its applications was not always easy to predict, even for its pioneers. For instance, the famous Ernest Rutherford, who discovered the atomic nucleus, once wrote: "Anyone who expects a source of power from the transformation of atoms is talking moonshine."

Progress in the physics of elementary interactions is parallel to the evolution of the concept of elementary constituents of matter. We know today that atoms, with the characteristic length scale of order of $\mathcal{O}\left(10^{-10}\right) \mathrm{m}$, consist of an electron shell and an atomic nucleus composed of protons and neutrons. The latter are composed of quarks which, together with leptons (electrons, muons and tauons), are most likely the elementary constituents of matter, with the characteristic length scale of order of $\mathcal{O}\left(10^{-18}\right) \mathrm{m}$. All the hitherto observed interactions between them (except for the gravitational ones) are described by the theory (modestly) 
called the Standard Model of elementary interactions. However, anticipating the second part of this paper, we are not sure if quarks and leptons interact with only those forces that have so far been observed in various phenomena. In fact, we strongly doubt it and expect to see more types of interactions, still beyond our observation because they are of even shorter range than $\mathcal{O}\left(10^{-18}\right) \mathrm{m}$ and quickly die out at larger distances. Since the investigation of interactions between quarks and leptons at shorter and shorter distances requires higher and higher energies, we need more powerful accelerators to discover them. Moreover, there are strong hints that in addition to the matter we can observe and produce in accelerators, as described by the Standard Model, there exists another type of matter in the universe, called dark matter, with its own elementary constituents and forces.

After more than a hundred years of research, the chapter opened by the discovery of radioactivity is now closed. It is a great, beautiful synthesis of theoretical insight and experimental research, mutually stimulating each other. The only missing element, the Higgs particle, predicted by the Standard Model and necessary for its theoretical consistency, was discovered at CERN in 2012. The Standard Model is a quantum field theory in which the physical picture of the microworld is based on the duality of fields and particles which are quanta of the fields, like photons being the quanta of electromagnetic field. It is a mathematically consistent theory describing with a very high precision all available experimental data, up to the highest energies accessible in proton collisions at the LHC. It also successfully explains everything what happened in the universe later than $10^{-9} \mathrm{~s}$ after the Big Bang.

\section{Significance of discovery of Higgs particle}

The discovery of the Higgs particle confirms the Standard Model as a very successful theory of elementary interactions in the energy range up to $10^{12} \mathrm{eV}$ and is a fundamental discovery for at least three general reasons closely linked to the success of this theory. First of all, it confirms the fundamental role of symmetries as the underlying principle for understanding the elementary structure of matter. Secondly, it explains the origin of masses of elementary particles. There is also the third reason that will be discussed in the next section of this paper.

A symmetry in physics means, generally speaking, indistinguishability. There are well known examples of symmetries of physical systems in the space, like, e.g., the symmetry under reflection or under a rotation around some axis. In the microworld, the concept of symmetry even has a deeper meaning. For instance, a proton and a neutron are different particles because they have different electric charges and (slightly) different masses

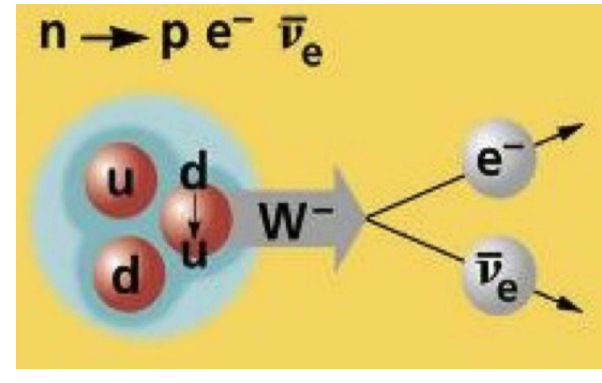

Fig. 1. The $\beta$ decay.

but their strong interactions are the same. They are indistinguishable with respect to strong interactions. The change of protons into neutrons and vice versa is the symmetry of strong interactions. The basis for understanding the phenomena for which elementary interactions are responsible is the search for certain symmetries among quarks and leptons and the conservation laws related to them. The unified theory of weak and electromagnetic interactions is a beautiful example of this principle and the Higgs particle is its very strong confirmation. Remember that in the quantum field theory, particles interact by emission (exchange) of a particle. The radioactive $\beta$ decay is similar to the electromagnetic interactions. In the latter, it is the exchange of a photon between two electrically charged particles which is responsible for their interaction. It was a long way for experimental and theoretical research to understand that the messengers of the weak interactions, responsible for $\beta$ decay, are very heavy particles called W bosons (see Fig. 1), eighty times heavier than a proton and discovered experimentally at CERN about 30 years ago.

Despite the fact that a photon is so different in mass (and also the electric charge) from a W boson, both types of interactions can be understood within the framework of a common ("unified") theory, called the Standard Model, based on a principle of certain symmetry. That extraordinary synthesis has been proposed by Abdus Salam and Steven Weinberg, combining earlier ideas of Sheldon Glashow and Robert Brout, Francois Englert and Peter Higgs.

How to reconcile so different masses (and the electric charges) of those particles with their description within one theory? Can there be any symmetry underlying such a theory? Obviously, it does not manifest itself at the level of the observed particles but perhaps it underlies the mathematical structure of the theory and gives some other experimentally testable predictions? Yes, the existence of the Higgs particle is the manifestation of that symmetry!

At the same time, the discovery of the Higgs particle confirms earlier theoretical ideas on the origin of mass of elementary constituents of matter. In fact, the underlying symmetry principle of the Standard Model and the origin of mass are very strongly linked together. The mass - the measure 
of inertia introduced by Newton - of a composite object is easy to explain as a "sum" of the constituent masses and their kinetic energies. But where does the mass of an elementary object come from?

It was a brilliant idea of Brout, Englert and Higgs, inspired by certain phenomena in condense matter physics, that the origin of elementary particle masses is their interaction with the "vacuum"!

The "vacuum" is not "empty": it is a physical medium that can interact with particles. Abdus Salam and Steven Weinberg used those ideas a few years later to construct the unified theory of electromagnetic and weak interactions. The "vacuum" is filled with the Higgs field, it can interact weakly but not electromagnetically. The interaction with the "vacuum" is responsible for the mass of a W particle but a photon remains massless. The same mechanism is the source of mass of other elementary particles. The "trace" of the existence of such a "vacuum" is a physical particle - called the Higgs particle with very specific properties confirmed with high precision by experiment. Thus, the discovery of the Higgs particle is the end of a certain story... the triumph of the worldview based on the principles of symmetry, with the "vacuum" interacting with particles and responsible for their masses.

\section{Does Standard Model aspire to be Theory of Everything?}

Most likely, it is not the case and here we arrive at the third general reason of the fundamental significance of the Higgs discovery.

It turns out again that we can make progress in our understanding of nature thanks to approximate laws of physics that describe - to a good approximation - a certain range of phenomena. This was the case with Newton's theory which approximates Einstein's more fundamental theory of relativity. This also holds true for the theory of electromagnetic phenomena which we understand today as an approximation to the Standard Model. And the same will probably happen to the Standard Model which will eventually turn out to be an approximation to some deeper theory.

However, it is intriguing that from time to time we may take a rest from that scientific climb. We can rest when a certain class of phenomena can be predicted and understood in a theoretically consistent way (it is important to stress the theoretical consistency and predictability) and successfully tested experimentally within the available experimental accuracy.

A perfect example is provided by quantum electrodynamics which describes electron and muonphoton interactions with incredible precision. For many decades, it has been successfully tested experimentally. It is a (nearly) perfect, fully consistent theory with no obvious need for its extension. However, we know today that it is just a low energy approximation to the Standard Model and this has now been experimentally confirmed by even more precise measurements of the electromagnetic properties of electrons and muons.

The Higgs particle is indispensable for the full mathematical consistency of the Standard Model. As the quantum electrodynamics for low energy electromagnetic interactions, it gives predictions for electroweak processes with arbitrarily high mathematical precision. And as it is the case with quantum electrodynamics, it is almost a perfect mathematical theory with no obvious need for its extension (see the next section, though). However, it does not mean that those predictions will remain correct once the experimental accuracy is improved even further.

Interestingly enough, the measurements of the properties of the Higgs particle, which confirm with high accuracy the Standard Model as the theory correctly describing all experimental data for elementary processes available at present, are likely to show the need for its extension once the experimental precision is even higher. The story with quantum electrodynamics will repeat itself. . .

\section{What next?}

There are some hints that the validity of the Standard Model has some limits. One of them is of a theoretical nature. There is a subtle difference between quantum electrodynamics and the Standard Model considered as the effective theories approximating a deeper one. It is a fact that the Standard Model has in its spectrum a spin zero particle, the Higgs particle. This is the first known elementary particle with spin zero. So far, nature has not seemed to like elementary spin zero particles. And indeed, an elementary spin zero particle leads to certain puzzles in the theoretical structure of the Standard Model, known under the name of the hierarchy problem.

Putting aside theoretical puzzles, there are also strong empirical hints. One of them is the fact that neutrinos have masses, whereas they enter into the Standard Model as massless particles. The other empirical hints come from cosmology and astrophysics. It is a well-established empirical fact that the universe is expanding. In remote past, it was small, densely packed with matter, and very hot. The matter particles were colliding with each other with very high energies determined by the temperature of the universe. The processes taking place between them at that time were a consequence of the laws of the physics of elementary interactions. The elementary constituents of matter discovered so far and their interactions described by the Standard Model explain only a part of the evolution of the universe (the formation of atomic nuclei and then atoms).

However, astrophysical observations tell us that there is very little of antimatter in our universe and, most likely, it is dominated by an unknown type of 
matter (dark matter) and unknown type of energy (dark energy). The processes responsible for such a structure of the universe must have occured at even higher temperatures or, equivalently, higher particle energies than those at which the validity of the Standard Model has been tested so far at the accelerators. Thus, we expect that at higher energies, new interactions and new forms of matter will manifest themselves as the building blocks of a deeper theory than the Standard Model of elementary interactions.

There are several interesting theoretical ideas on embedding the Standard Model into a deeper theory, without destroying its successful predictions and addressing the challenges mentioned above. The most promising theoretical self-consistent hypotheses are based on new symmetries (supersymmetry and supergravity unifying gravity with other interactions) and on the existence of additional, very small dimensions of space. Does the space have only three dimensions? Or, perhaps, we live in more space dimensions, with very small additional dimensions? How to "see" them, if they are really there? Can we see their signatures in the accelerators? Did they play any role in the evolution of the universe from the Big Bang to the present time? How strong can be their impact on the gravitational forces? Maybe the laws of Newton and Einstein need to be improved? Another interesting hypothesis is the existence of invisible forms of matter (a hidden world) interacting with new strong interactions and with their own elementary constituents.

A quest for answers to those questions is the main goal of the present experimental and theoretical research worldwide and of research planned for the future in the field of the physics of elementary interactions. And, as it has been going on for seventy years now, the Polish scientists participate in that research with great success!

\section{Acknowledgments}

I warmly thank Helena Białkowska for her contribution to this text. 